Condensed Matter Physics, 2004, Vol. 7, No. 2(38), pp. 331-346

\title{
Transport properties of the strongly correlated systems
}

\author{
T.Domański, M.Krawiec, M.Michalik, K.I.Wysokiński \\ Institute of Physics, M. Curie Skłodowska University, \\ 20-031 Lublin, Poland
}

Received March 12, 2004, in final form May 6, 2004

\begin{abstract}
The transport properties of various systems are studied here in the context of three different models. These are: (i) the disordered Hubbard model applicable to correlated binary alloys with a general disorder, (ii) the Anderson model used in describing the Kondo physics of a quantum dot connected to the external superconducting leads, and (iii) the Ranninger-Robaszkiewicz model applied to the study of optical properties of the system with preformed electron pairs above the temperature of transition to the superconducting state. We calculate the density of states, specific heat, the Wilson ratio and conductivity of the correlated binary alloy with off-diagonal disorder. We investigate the conditions under which the Kondo peak appears in the density of states and in the conductance of a dot coupled to the external superconducting leads. We analyze the effect of the pseudogap on the optical spectra in the high temperature superconductors described by the boson-fermion model.
\end{abstract}

Key words: coherent potential approximation, slave bosons, non-equilibrium transport, quantum dot, continuous transformation, pseudogap

PACS: 71.10.-w, 72.10.-d, 73.23.-b, 74.20.-z

\section{Introduction}

The present paper discusses some issues relevant to very important issues of the modern condensed matter physics. These are related to the study of thermodynamic and transport properties of disordered alloys, the non-equilibrium transport of quantum dots between the external leads as well as some aspects of the high temperature superconductivity.

A number of heavy fermion and other correlated systems have been found with the general chemical structure in the form of $\mathrm{MA}_{x} \mathrm{~B}_{1-x}$. To describe their properties it is vital to take into account the correlated character of carriers and the presence of disorder. The interplay of disorder and the weak Coulomb interactions is of great importance in the description of numerous properties of real systems. The subject has 
been studied from a viewpoint of the weak coupling theory [1] and it has been found that renormalization procedure flows into the strong coupling regime. Therefor, we start here from the strong correlation limit.

Coulomb interactions are also of primary importance in nano- and mesoscopic systems which recently have become the subject of many studies due to their unusual properties and foreseeable technological applications. The quantum dot we study in section 3 is an example of such a system. Its importance lies both in the basic and in the applied research. It is an example of quantum impurity [2] with the controllable parameters. It permits to study the many body interactions in equilibrium and nonequilibrium conditions [3]. The recent proposals [4] to use quantum dots as building blocks of qubits (i.e., the elementary units of information in quantum computers) together with the predicable control of their decoherence by the external voltage [5] makes the subject even more interesting and worth studying.

The pseudogap phase of high temperature superconductors certainly belongs to the most puzzling behaviors ever observed in the condensed matter physics. The successes [6] of the boson fermion model, introduced by Ranninger and Robaszkiewicz and later studied by others [7] in elucidating important aspects of the pseudogap phase have encouraged us to look at its signatures in the transport properties. We have found strong effect of the pseudogap on the optical spectra above $T_{\mathrm{c}}$.

\section{Strongly correlated alloys with off-diagonal disorder}

Let us assume that disordered and correlated alloy can be adequately described by the following Hubbard Hamiltonian

$$
H=\sum_{i j \sigma} t_{i j} c_{i \sigma}^{+} c_{j \sigma}+\sum_{i} U_{i} c_{i \downarrow}^{+} c_{i \downarrow} c_{i \uparrow}^{+} c_{i \uparrow}+\sum_{i \sigma}\left(\epsilon_{i}-\mu\right) c_{i \sigma}^{+} c_{i \sigma}
$$

Here $t_{i j}$ denote the hopping amplitudes for an electron to hop from site $i$ to $j$ or vice versa, $c_{i \sigma}^{+}\left(c_{i \sigma}\right)$ is the creation (annihilation) operator of a spin $\sigma$ electron at site $i, \mu$ is a chemical potential and $U$ denotes the energy cost when two electrons occupy the same site. The site energies $\epsilon_{i}$ take on two values $\epsilon_{A}$ or $\epsilon_{B}$ when site $i$ is occupied by an $A$ or $B$ type electron i.e., we assume that the ideal crystal lattice is occupied at random by two types of atoms: $A$ with probability $x$ and $B$ with probability $(1-x)$. Accordingly, the hopping integrals take on three values $t_{A A}, t_{A B}=t_{B A}$ and $t_{B B}$.

To treat the many body interactions of (1) the slave boson technique has been introduced by Kotliar and Rückenstein [8] and used inter alia by Lavagna [9] to study the Mott metal-insulator transition in clean system. To calculate the averages over the disorder we use the coherent potential approximation (CPA) in its general version [10] capable of treating the diagonal (random site energies) and off-diagonal disorder (random hopping integrals). The present work generalizes our previous study $[11,12]$ of impure correlated materials. The general strategy is similar to that presented in [12]. Instead of the electron operators $c_{i \sigma}^{+}, c_{i \sigma}$ one introduces new fermion $a_{i \sigma}\left(a_{i \sigma}^{+}\right)$and auxiliary boson creation $e_{i}^{+}, s_{i \sigma}^{+}, d_{i}^{+}$and annihilation $e_{i}, s_{i \sigma}, d_{i}$ operators. They take 
care of unoccupied states $e_{i}^{+} e_{i}$, singly occupied $s_{i \sigma}^{+} s_{i \sigma}$ and doubly occupied states $d_{i}^{+} d_{i}$. Replacement is done according to the following scheme

$$
\begin{aligned}
|\uparrow\rangle_{i} & =c_{i \uparrow}^{+}|0\rangle \rightarrow s_{i \uparrow}^{+} e_{i} a_{i \uparrow}^{+}|v a c\rangle, \\
|\downarrow\rangle_{i} & =c_{i \downarrow}^{+}|0\rangle \rightarrow s_{i \downarrow}^{+} e_{i} a_{i \downarrow}^{+}|v a c\rangle, \\
|\uparrow \downarrow\rangle_{i} & =c_{i \uparrow}^{+} c_{i \downarrow}^{+}|0\rangle \rightarrow d_{i}^{+} e_{i} a_{i \uparrow}^{+} a_{i \downarrow}^{+}|v a c\rangle .
\end{aligned}
$$

Additional conditions have to be applied to the boson fields as well as a new fermion operator to guarantee a conservation of the number of physically accepted states at a given site $i$, which can be either empty, singly or doubly occupied

$$
e_{i}^{+} e_{i}+\sum_{\sigma} s_{i \sigma}^{+} s_{i \sigma}+d_{i}^{+} d_{i}=1
$$

and the conservation of charge

$$
a_{i \sigma}^{+} a_{i \sigma}=s_{i \sigma}^{+} s_{i \sigma}+d_{i}^{+} d_{i}
$$

In the mean field approximation, boson operators are replaced by the numbers. However, in the disordered system we expect that these numbers will get the random values. To take these constraints into account one introduces the Lagrange multipliers $\lambda_{i \sigma}^{(1)}$ and $\lambda_{i \sigma}^{(2)}$ and the resulting model contains a disorder without interactions

$$
H_{\mathrm{MF}}=\sum_{i j \sigma} t_{i j} \xi_{i \sigma} \xi_{j \sigma} a_{i \sigma}^{+} a_{j \sigma}+\sum_{i \sigma}\left(\lambda_{i \sigma}^{(2)}+\epsilon_{i}-\mu\right) a_{i \sigma}^{+} a_{i \sigma}+\text { const }
$$

Following [12] we minimize the energy of the system and find equations for the random values of auxiliary fields. Application of the CPA [10] permits us to solve the disordered problem and find the averaged and conditionally averaged Green functions. The details will be presented elsewhere [13]. This enables us to calculate thermodynamic and transport properties of the system. In particular, in figure 1 we show the density of states of a correlated alloy with diagonal disorder for three values of the Hubbard interaction $U$. Note the peak in the density of states emerging at low energies with an increasing disorder. This behavior has been observed previously [11] and discussed at length therein. For numerical purposes we consider the cubic lattice with the tight binding dispersion

$$
\epsilon_{\mathbf{k}}=-\frac{W}{3}\left(\cos k_{x}+\cos k_{y}+\cos k_{z}\right),
$$

where $W=6 t$ is the bandwidth and $t$ is the hopping integral.

The electron specific heat $c_{\mathrm{e}}$ shown in figure 2 has been calculated from the following formula

$$
c_{\mathrm{e}}=2 \int \mathrm{d} \omega D(\omega) \omega \frac{\mathrm{d}}{\mathrm{d} T} f(\omega, T)
$$




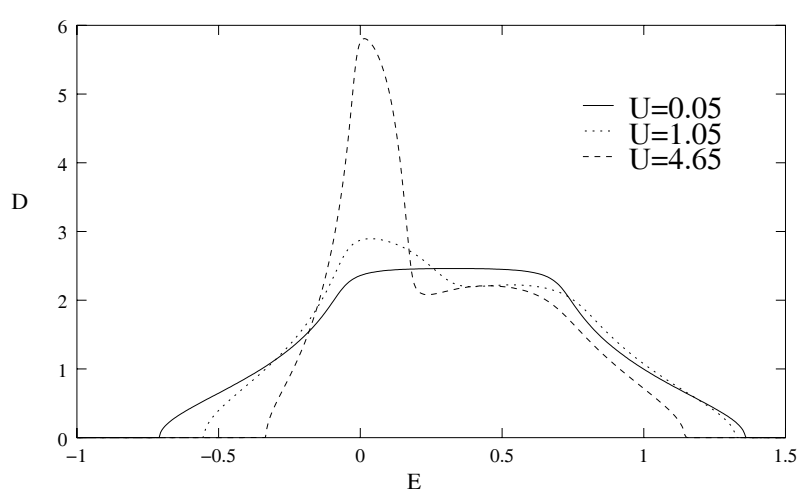

Figure 1. Energy dependence of the density of states $D$ for a binary alloy $A_{x} B_{1-x}$ with $x=0.5, \delta \equiv \varepsilon_{A}-\varepsilon_{B}=0.5 \mathrm{~W}$ and electron concentration per lattice site $n=0.5$. Values of the Coulomb interaction $U$ (expressed in units of $W$ ) are indicated in the legend.

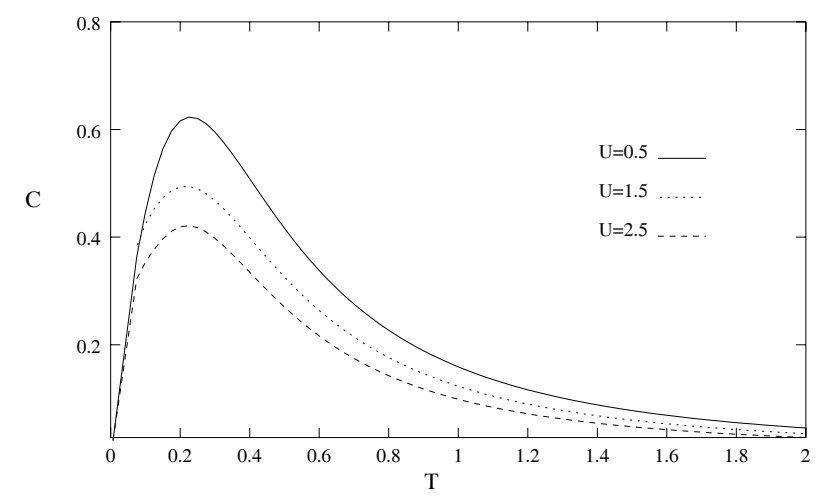

Figure 2. The specific heat $c$ as a function of temperature $T$ (in units of $W / k_{\mathrm{B}}$ ) for $x=0.5, n=0.5, \delta=0.2 \mathrm{~W}$ and several values of the Coulomb interaction $U$.

where $f(\omega, T)$ is the Fermi function at temperature $T$ and $D(\omega)=-1 / \pi \operatorname{Im}\langle G\rangle$ is the alloy density of states. The $\mathrm{d} c$ conductivity has been calculated from the KuboGreenwood formula averaged over the disorder with CPA. As usually the vertex corrections have been neglected in a spirit of the single site theory. Again, it is shown in figure 3 as a function of $U$. The non-monotonic dependence indicates a subtle interplay of the disorder and interactions. This issue will be discussed in depth elsewhere [13].

\section{Transport through Quantum Impurity Coupled to the Superconducting Leads}

The study of quantum dots, both experimental and theoretical has been extensively continued over the last two decades or so [14-23]. Here we propose to calculate transport properties of the quantum dot $(Q D)$ connected to the superconducting leads $(S-Q D-S$ junction). It has to be noted that in this case, besides single particle tunnelling, the Andreev reflection processes [24] contribute to the transport 


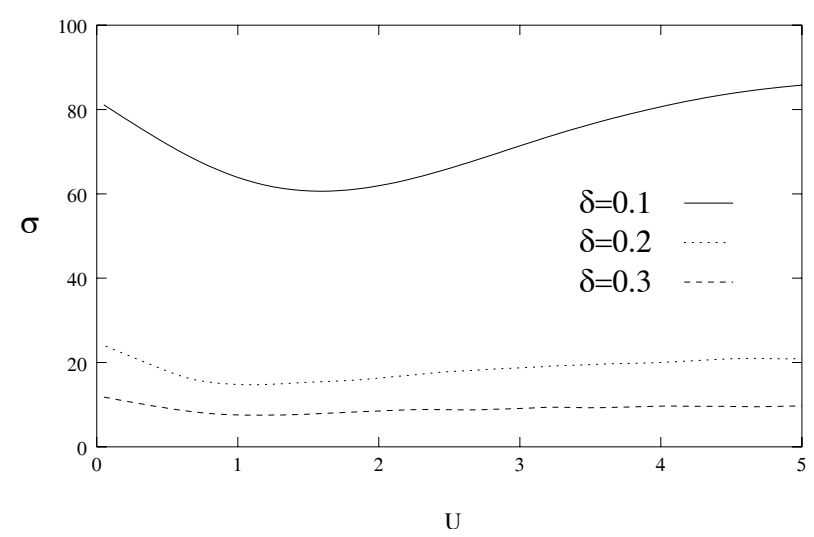

Figure 3. $D c$ conductivity of the correlated alloy as a function of Coulomb interaction $U$ for $x=0.5, n=0.5$ and several values $\delta$ expressed in units of $W$.

of the system. The Andreev scattering, during which an electron impinging on the normal metal-superconductor interface is reflected back as a hole, has been shown to play a crucial role in the transport in various hybrid mesoscopic superconductivity devices [25].

Such quantum dot systems have been studied theoretically [26-34] but there exist very limited experimental data on the subject $[35,36]$. Theoretical studies $[26-34]$ of the current in the $S-Q D-S$ junctions have been mainly restricted to the case of classical superconductors with $s$-wave order parameter (see, however, [30]). Here we shall be particularly interested in the studies of the transport via quantum dot coupled to the electrodes made of (the same or different) exotic superconductors with $d$-wave symmetry of the order parameter.

The purpose of the present work is to study the electron transport through the quantum dot coupled to the superconducting leads with various symmetries of the superconducting order parameter. The dot is described by the Anderson impurity model in the limit of the strong on-dot correlations $(U \rightarrow \infty)$. Due to large $U$, in the first approximation, one can neglect the Andreev reflections [28] and deal only with the single particle tunnelling. The effect of the Andreev processes on the tunnelling current through $S-Q D-S$ structures is left for the future publication.

In the limit of the strong on-dot correlations $(U \rightarrow \infty)$ the Anderson Hamiltonian [37] of the $S-Q D-S$ system can be written in the slave boson representation $[38,39]$ as

$$
\begin{aligned}
H= & \sum_{\lambda \mathbf{k} \sigma} \epsilon_{\lambda \mathbf{k}} c_{\lambda \mathbf{k} \sigma}^{+} c_{\lambda \mathbf{k} \sigma}+\sum_{\lambda \mathbf{k}}\left(\Delta_{\lambda \mathbf{k}} c_{\lambda \mathbf{k} \uparrow}^{+} c_{\lambda-\mathbf{k} \downarrow}^{+}+\text {c.c. }\right)+E_{\mathrm{d}} \sum_{\sigma} f_{\sigma}^{+} f_{\sigma} \\
& +\sum_{\lambda \mathbf{k} \sigma} V_{\lambda \mathbf{k}}\left(c_{\lambda \mathbf{k} \sigma}^{+} b^{+} f_{\sigma}+\text { h.c. }\right) .
\end{aligned}
$$

$\lambda=L, R$ denotes here the left $(L)$ or right $(R)$ lead of the system. Other parameters have the following meaning: $c_{\lambda \mathbf{k} \sigma}^{+}\left(c_{\lambda \mathbf{k} \sigma}\right)$ denotes a creation (annihilation) operator for a conduction electron with the wave vector $\mathbf{k}$, spin $\sigma$ in the lead $\lambda, \Delta_{\lambda \mathbf{k}}$ is the superconducting order parameter in the lead $\lambda$, and $V_{\lambda \mathbf{k}}$ is the hybridization matrix 
element between conduction electron of energy $\varepsilon_{\lambda \mathbf{k}}$ in the lead $\lambda$ and the localized electron on the dot with the energy $E_{\mathrm{d}} \cdot f_{\sigma}^{+}\left(f_{\sigma}\right)$ is the fermion operator which denotes the singly occupied state on the dot, while $b^{+}(b)$ stands for empty state on the dot. Operator of the physical electron can be expressed by the product of the fermion and boson operators [38,39].

When the Andreev reflections are neglected, the tunnelling current in the $S-$ $Q D-S$ system can be expressed in terms of the single particle spectral density of states of the dot [28]. The current flowing out of the lead $\lambda$ is written by

$$
J_{\lambda}=\frac{e}{\hbar} \sum_{\mathbf{k} \sigma} V_{\lambda \mathbf{k}} \int_{-\infty}^{\infty} \frac{\mathrm{d} \omega}{2 \pi}\left(G_{\mathrm{d}, \lambda \mathbf{k} \sigma}^{<}(\omega)-G_{\lambda \mathbf{k} \sigma, \mathrm{d}}^{<}(\omega)\right),
$$

where $G_{\mathrm{d}, \lambda \mathbf{k} \sigma}^{<}(\omega)$ is the Fourier transform of the Keldysh Green function [40] $G_{\mathrm{d}, \lambda \mathbf{k} \sigma}^{<}(t)=\mathrm{i}\left\langle c_{\lambda \mathbf{k} \sigma}^{+}(0) b^{+}(t) f_{\sigma}(t)\right\rangle$. Using the equation of motion technique for the nonequilibrium Green functions [41] and making approximations due to Kang [28] we obtain the single particle current in the form

$$
J_{s p}=\frac{2 e}{\hbar} \sum_{\sigma} \int_{-\infty}^{\infty} \mathrm{d} \omega \tilde{\Gamma}^{S}(\omega)\left[f_{\mathrm{L}}(\omega)-f_{\mathrm{R}}(\omega)\right]\left(-\frac{1}{\pi}\right) \operatorname{Im}\left[G_{\sigma}^{r}(\omega)\right]_{11},
$$

where $f_{\lambda}=\left[\mathrm{e}^{\beta\left(\omega-\mu_{\lambda}\right)}+1\right]^{-1}$ is the Fermi distribution function of the lead $\lambda, \tilde{\Gamma}^{S}(\omega)=$ $\left(\Gamma_{\mathrm{L}}^{S}(\omega) \Gamma_{\mathrm{R}}^{S}(\omega)\right) /\left(\Gamma_{\mathrm{L}}^{S}(\omega)+\Gamma_{\mathrm{R}}^{S}(\omega)\right)$ is the effective coupling of the quantum dot to the leads. To calculate $\widehat{G}_{\sigma}^{r}(\omega)$ we have used the modified slave boson approach $[42,43]$ and got

$$
\left[\omega \tau_{0}-E_{\mathrm{d}} \tau_{3}-\widehat{\Sigma}_{0}(\omega)-\widehat{\Sigma}_{I}(\omega)\right] \widehat{G}_{\sigma}^{r}(\omega)=\widehat{q}
$$

where $\tau_{0}$ and $\tau_{3}$ are the Pauli matrices. The noninteracting $\left(\widehat{\Sigma}_{0}\right)$ and interacting $\left(\widehat{\Sigma}_{I}\right)$ selfenergies and the matrix $\widehat{q}$ will not be reproduced herein. Instead of

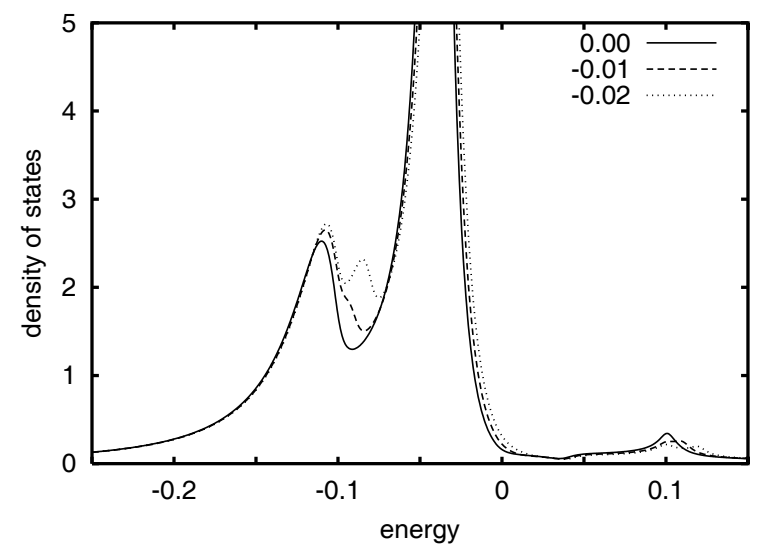

Figure 4. The density of states of the quantum dot connected to the $d$-wave superconductors for different values of the chemical potentials $\left(\mu_{\mathrm{L}}=-\mu_{\mathrm{R}}\right)$. Other parameters have the following values: $\Gamma_{\mathrm{L}}^{S}=\Gamma_{\mathrm{R}}^{s}=0.01, E_{\mathrm{d}}=-0.07, \Delta^{s}=0.1$ in units of the bandwidth $W$. 
this, the numerical results will be presented for the $s$ - and $d$-wave superconducting electrodes. For the $s$-wave ( $B C S$-like) superconductor the energy gap has a constant value $\left(\Delta_{\lambda \mathbf{k}}=\Delta_{\lambda}^{s}\right)$ (there is no dependence on the wave vector). In case of the $d$-wave superconductor we assume the order parameter in the following form: $\Delta_{\lambda \mathbf{k}}=\Delta_{\lambda}^{\mathrm{d}}\left[\cos \mathbf{k}_{x} a-\cos \mathbf{k}_{y} a\right]$, where $a$ is the lattice constant taken for simplicity as $a=1$. The equilibrium (solid line) and nonequilibrium (dashed and dotted lines) density of states of the quantum dot connected to the $d$-wave superconductors $(S C)$ is displayed in the figure 4.

It is worth noting that we deal now with the resonance in the DOS of the quantum dot, instead of the bound state, as for the $s$-wave superconductors. This is due to the finite $D O S$ (except the point $\omega=\mu$ ) of the $d$-wave superconductor. Position of the resonance weakly depends on the voltage $\left(\mu_{\mathrm{L}}-\mu_{\mathrm{R}}\right)$. We also observe the additional structure near the energy $\omega \approx \Delta^{\mathrm{d}}$, as in the case of the $B C S$-like $S C$.

Figure 5 refers to the situation in which one electrode is $s$-wave while another one is the $d$-wave superconductor with the same values of the $S C$ order parameters $\Delta^{s}=\Delta^{\mathrm{d}}=0.1 \mathrm{~W}$. The structure of the DOS is similar to the one shown in figure 4 . Now we also have a well resolved structure coming from the $s$-wave superconducting gap. Such a behavior is expected due to the structure of the selfenergy $\left(\widehat{\Sigma}_{0}+\widehat{\Sigma}_{I}\right)$. This is simply a sum of the densities of states for $s$ - and $d$-wave $S C$.

As we mentioned earlier the current through the quantum dot should have two components. One component is connected with the single charge tunnelling and the second one comes from the Andreev reflection processes [24]. However, in our system the on-dot Coulomb repulsion $U$ is assumed to be the largest parameter in the theory and is taken as infinitely large. So, at this condition Andreev processes are prohibited [28] and we have only the single particle component of the current.

The differential conductance of the quantum dot couple to the $d$-wave superconductors is displayed in figure 6 .

It is worthwhile noticing that the current does flow even for the $e V_{S D}<\Delta^{\mathrm{d}}$, which is due to a finite value of $D O S$ in the $d$-wave $S C$. The other features are

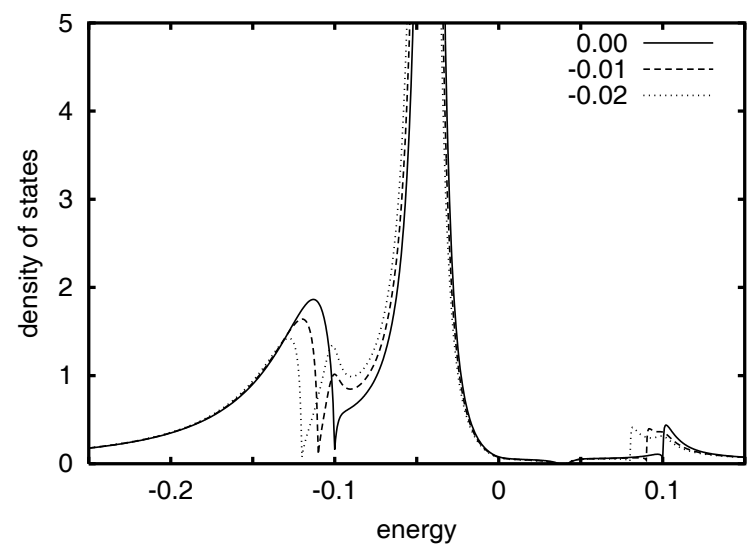

Figure 5. The same as in figure 4, but now one electrode is the $s$-wave while another one is $d$-wave superconductor. 


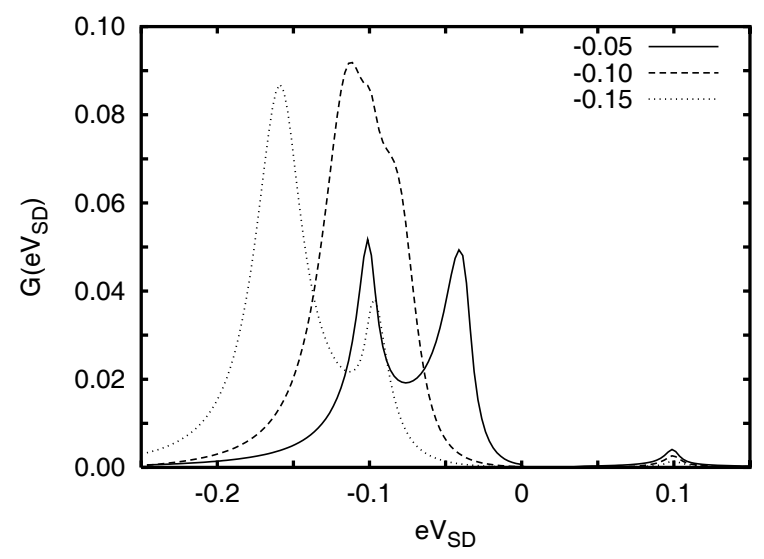

Figure 6. The single particle differential conductance in the $S-Q D-S$ system for various values of the dot's energy level $E_{\mathrm{d}}$. Both superconducting energy gaps are assumed to have $d$-wave symmetry and equal values $\Delta_{\mathrm{L}}^{\mathrm{d}}=\Delta_{\mathrm{R}}^{\mathrm{d}}=0.1 \mathrm{~W}$. The coupling constants are $\Gamma_{\mathrm{L}}^{S}=\Gamma_{\mathrm{R}}^{S}=0.01 W$, in units of the bandwidth $W$.

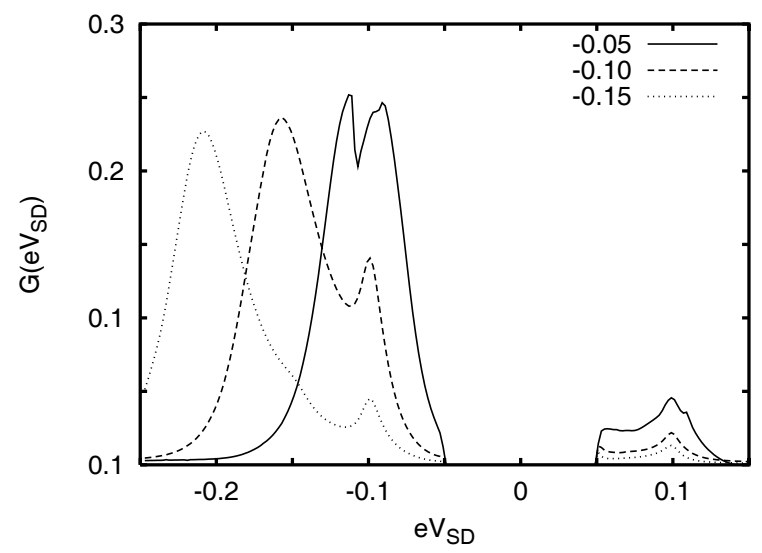

Figure 7. The same as in figure 6, but now one electrode is the $s$-wave and another one $d$-wave superconductors with $\Delta_{\mathrm{L}}^{\mathrm{d}}=\Delta_{\mathrm{R}}^{\mathrm{d}}=0.1 \mathrm{~W}$ and $\Gamma_{\mathrm{L}}^{S}=\Gamma_{\mathrm{R}}^{S}=0.01 \mathrm{~W}$.

similar to the case of the $B C S$-like superconductors. However, the main difference arises when the dot energy level $\left(E_{\mathrm{d}}\right)$ lies within the energy gap $\left(\Delta^{\mathrm{d}}\right)$. The broad resonance emerges at $\omega \approx E_{\mathrm{d}}$ (as in the $D O S$ ) and is due to the finite $d$-wave superconducting DOS.

The results obtained for the geometry with the quantum dot connected to one $s$ wave and the other $d$-wave superconductors (with the same values of $\Delta$ ) are depicted in figure 7 . The main features are similar to a junction with the $d$-wave superconductors. There is, however, a strong renormalization of the position of the resonance for $E_{\mathrm{d}}<\Delta$, so it is located near the $\omega=\Delta$ (solid line). 


\section{Optical conductivity in the pseudogap region}

One of the main theoretical issues presently widely debated in the literature concerns the pseudogap effect which has been observed experimentally above $T_{\mathrm{c}}$ in the underdoped and optimally doped samples of high temperature superconductors. Since the single particle electron states are (partly) washed out from the same parts of the Brillouin zone where, for $T<T_{\mathrm{c}}$, the true superconducting gap is formed, it is quite natural to interpret this phenomenon as a precursor of superconducting correlations. The pseudo- and superconducting gaps are assumed there to be related with some common order parameter. In pseudogap state, the order parameter is affected by the strong quantum fluctuations. Hence, the long range phase coherence cannot be established. Although there are also alternative interpretations of the pseudogap effect (in terms of the competing orders, microscopic currents, etc.) it seems that the recent experimental facts [47] work in favor of the precursor scenario.

In this section we want to address a question whether the pseudogap (treated as a precursor of superconducting ordering) affects qualitatively the charge response of the system. For this purpose we investigate the two component model in which the single particle charge carriers (fermions) are assumed to coexist and interact with the preformed pairs (the hard-core bosons). Hamiltonian of such boson fermion model is given by

$$
H=\sum_{\mathbf{k}, \sigma}\left(\varepsilon_{\mathbf{k}}-\mu\right) c_{\mathbf{k} \sigma}^{\dagger} c_{\mathbf{k} \sigma}+\sum_{\mathbf{q}}\left(E_{\mathbf{q}}-2 \mu\right) b_{\mathbf{q}}^{\dagger} b_{\mathbf{q}}+\frac{1}{\sqrt{N}} \sum_{\mathbf{k}, \mathbf{p}} v\left(b_{\mathbf{p}+\mathbf{k}}^{\dagger} c_{\mathbf{k} \downarrow} c_{\mathbf{p} \uparrow}+\text { h.c. }\right) \text {. }
$$

Its relevance to the high $T_{\mathrm{c}}$ superconductivity as well as the precursor type features have been discussed in a number of papers [7].

Let us suppose that this two component system (12) reacts to the ac electric field $\mathbf{E}(\mathbf{r}, t)$. Within the linear response theory, the induced current $\mathbf{j}(\mathbf{r}, t)$ is expected to scale linearly with a perturbation. Fourier transform of the current density can be written as

$$
j_{\alpha}(\mathbf{q}, \omega)=\sum_{\beta} \sigma_{\alpha, \beta}(\mathbf{q}, \omega) E_{\beta}(\mathbf{q}, \omega)
$$

where $\alpha, \beta$ stand for the Cartesian coordinates. The complex conductivity $\sigma(\mathbf{q}, \omega)$ can be determined using the Kubo formula [48] which in the case of the two component system (12) is given by

$$
\sigma_{\alpha, \beta}(\mathbf{q}, \omega)=\frac{\mathrm{i}}{\omega}\left[\left(\frac{e^{2} n^{\mathrm{F}}}{m^{\mathrm{F}}}+\frac{4 e^{2} n^{\mathrm{B}}}{m^{\mathrm{B}}}\right) \delta_{\alpha, \beta}+\Pi_{\alpha, \beta}(\mathbf{q}, \omega)\right] .
$$

$n^{\mathrm{F}}$ and $n^{\mathrm{B}}$ denote the concentration of fermions (carrying the elementary charge e) and bosons (of the double charge 2e). The retarded current-current correlation function $\Pi_{\alpha, \beta}(\mathbf{q}, \omega)$ appearing in (14) is defined in a standard way

$$
\Pi_{\alpha, \beta}(\mathbf{q}, \omega)=-\int_{0}^{1 / k_{\mathrm{B}} T} \mathrm{~d} \tau e^{\omega \tau}\left\langle T_{\tau} j_{\alpha}(\mathbf{q}, \tau) j_{\beta}(-\mathbf{q}, 0)\right\rangle
$$


where the total current operator is composed correspondingly of the fermion and boson contributions $\mathbf{j}=\mathbf{j}^{\mathrm{F}}+\mathbf{j}^{\mathrm{B}}$

$$
\begin{aligned}
\mathbf{j}^{\mathrm{F}}(\mathbf{q}, \tau) & =e \sum_{\mathbf{k}, \sigma} \nabla \varepsilon_{\mathbf{k}+\mathbf{q} / 2} c_{\mathbf{k} \sigma}^{\dagger}(\tau) c_{\mathbf{k}+\mathbf{q} \sigma}(\tau), \\
\mathbf{j}^{\mathrm{B}}(\mathbf{q}, \tau) & =2 e \sum_{\mathbf{k}} \nabla E_{\mathbf{k}+\mathbf{q} / 2} b_{\mathbf{k}}^{\dagger}(\tau) b_{\mathbf{k}+\mathbf{q}}(\tau) .
\end{aligned}
$$

In order to determine the paramagnetic part of the conductivity (14) which is given there by $\Pi_{\alpha, \beta}(\mathbf{q}, \omega)$ one has to calculate the two-particle Green function (15). Within the lowest order perturbation theory it can be estimated from the bubble diagram, which effectively represents a convolution of the single particle fermion or boson propagators. Many body effects are included on this level of approximation through the quasiparticle damping.

The proper description of transport requires vertex corrections to be taken into account[48]. However, for the ordered phases (such as superconducting, ferromagnetic, etc.) it is usually fairly sufficient to work with the lowest order estimation of $\Pi_{\alpha, \beta}(\mathbf{q}, \omega)$. The main effect of interactions comes there due to the gapped structure of the single particle excitations. In the pseudogap phase we deal with a short range ordering leads to the partial suppression of the low lying single particle states. We can thus use the lowest order estimate for $\Pi_{\alpha, \beta}(\mathbf{q}, \omega)$ at least as a starting point of our discussion.

The current-current correlation function (15) consists of the following four terms $\Pi=\Pi^{\mathrm{F}, \mathrm{F}}+\Pi^{\mathrm{B}, \mathrm{B}}+\Pi^{\mathrm{F}, \mathrm{B}}+\Pi^{\mathrm{B}, \mathrm{F}}$ which correspond to fermion-fermion, boson-boson and the mixed current correlation functions. In the normal phase $T>T_{\mathrm{c}}$ a main contribution to the charge transport comes from the first two terms. The other mixed correlation functions play a considerable role only in the superconducting state as discussed in [49]. Using the above mentioned approximations we can express the correlation functions via

$$
\begin{aligned}
& \Pi_{\alpha, \beta}^{\mathrm{F}, \mathrm{F}}(\mathbf{q}, \omega)=e^{2} \sum_{\mathbf{k}, \sigma} \frac{\partial \tilde{\varepsilon}_{\mathbf{k}+\mathbf{q} / 2}}{\partial k_{\alpha}} \frac{\partial \tilde{\varepsilon}_{\mathbf{k}+\mathbf{q} / 2}}{\partial k_{\beta}} \\
& \times \int \mathrm{d} \omega_{1} A^{\mathrm{F}}\left(\mathbf{k}, \omega_{1}\right) \int \mathrm{d} \omega_{2} A^{\mathrm{F}}\left(\mathbf{k}+\mathbf{q}, \omega_{2}\right) \frac{f\left(\omega_{1}, T\right)-f\left(\omega_{2}, T\right)}{\omega+\omega_{1}-\omega_{2}}(18 \\
& \Pi_{\alpha, \beta}^{\mathrm{B}, \mathrm{B}}(\mathbf{q}, \omega)=4 e^{2} \sum_{\mathbf{k}, \sigma} \frac{\partial \tilde{E}_{\mathbf{k}+\mathbf{q} / 2}}{\partial k_{\alpha}} \frac{\partial \tilde{E}_{\mathbf{k}+\mathbf{q} / 2}}{\partial k_{\beta}} \\
& \times \int \mathrm{d} \omega_{1} A^{\mathrm{B}}\left(\mathbf{k}, \omega_{1}\right) \int \mathrm{d} \omega_{2} A^{\mathrm{B}}\left(\mathbf{k}+\mathbf{q}, \omega_{2}\right) \frac{b\left(\omega_{1}, T\right)-b\left(\omega_{2}, T\right)}{\omega+\omega_{1}-\omega_{2}},
\end{aligned}
$$

where $f(x, t)$ and $b(x, T)$ stand for the Fermi-Dirac and Bose-Einstein distribution functions. Moreover, we introduced the spectral functions $A^{F(B)}(\mathbf{q}, \omega)=-\pi^{-1}$ $\operatorname{Im}\left\{G^{F(B)}\left(\mathbf{q}, \omega+i 0^{+}\right)\right\}$of the single particle fermion $G^{\mathrm{F}}(\mathbf{q}, \tau)=-\left\langle T_{\tau} c_{\mathbf{q} \sigma}(\tau) c_{\mathbf{q}, \sigma}^{\dagger}\right\rangle$ and boson Green function $G^{\mathrm{B}}(\mathbf{q}, \tau)=-\left\langle T_{\tau} b_{\mathbf{q}}(\tau) b_{\mathbf{q}}^{\dagger}\right\rangle$.

The fermion and boson excitation spectra are found using the canonically transformed Hamiltonian $H(l)=e^{S(l)} H e^{-S(l)}$ [50], where upon a suitable choice of 


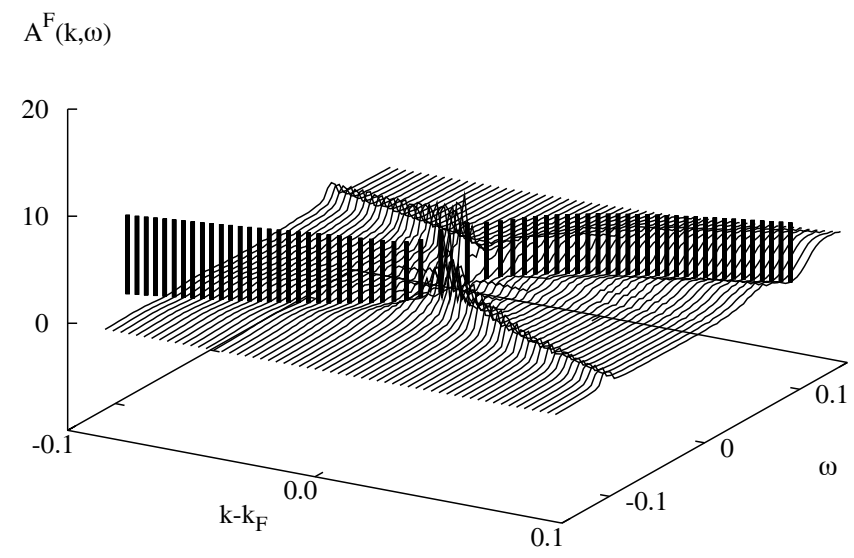

Figure 8. The single particle spectral function $A^{\mathrm{F}}(\mathbf{k}, \omega)$ in a neighborhood of the Fermi surface $\mathbf{k} \sim \mathbf{k}_{\mathrm{F}}$ for $T=0.007 \mathrm{~W} / k_{\mathrm{B}}$ corresponding to the temperature region $T^{*}>T>T_{\mathrm{c}}$. Note a pseudogap feature in the dispersion of the long-lived fermion states and the appearance of the additional Bogoliubov shadow branch around $\omega=-\tilde{\varepsilon}_{\mathbf{k}}$. Energy $\omega$ is expressed in units of the fermion bandwidth $W$, spectral function through $W^{-1}$ and momentum $k-k_{\mathrm{F}}$ in units of the inverse lattice constant.

$S(l)$ in the limit $l \rightarrow \infty$ the subsystems become disentangled one from another. To some extent such a transformation resembles the numerical renormalization group procedure with the cut-off $\Lambda=1 / \sqrt{l}$ [51]. Effective energies are renormalized to: $\varepsilon_{\mathbf{k}}-\mu \longrightarrow \tilde{\varepsilon}_{\mathbf{k}}, E_{\mathbf{q}}-2 \mu \longrightarrow \tilde{E}_{\mathbf{q}}$ and $v \rightarrow 0$. For the temperature regime $T^{*}>T>T_{\mathrm{c}}$, the resulting fermion spectrum $\tilde{\varepsilon}_{\mathbf{k}}$ shows up the asymmetric pseudogap structure near the Fermi momentum $\mathbf{k}_{\mathrm{F}}$ as discussed in [50]. The same procedure applied to the calculation of the single particle Green functions gives for $T>T_{\mathrm{c}}$ the following fermion spectral function $[6] A^{\mathrm{F}}(\mathbf{k}, \omega)=$ $\left|\tilde{P}_{\mathbf{k}}\right|^{2} \delta\left(\omega-\tilde{\varepsilon}_{\mathbf{k}}\right)+\frac{1}{N} \sum_{\mathbf{q}}\left[b\left(\tilde{E}_{\mathbf{q}}\right)+f\left(\tilde{\varepsilon}_{\mathbf{q}-\mathbf{k}}\right)\right]\left|\tilde{r}_{\mathbf{k}, \mathbf{q}}\right|^{2} \delta\left(\omega-\tilde{E}_{\mathbf{q}}+\tilde{\varepsilon}_{\mathbf{q}-\mathbf{k}}\right)$. Coefficients $\tilde{P}_{\mathbf{k}}$ and $\tilde{r}_{\mathbf{k}, \mathbf{q}}$ are correspondingly given by the renormalization equations in [6]. We notice that $A^{\mathrm{F}}(\mathbf{k}, \omega)$ describes the long-lived quasiparticles of energy $\tilde{\varepsilon}_{\mathbf{k}}$ and the remaining part of the spectral weight $1-\left|\tilde{P}_{\mathbf{k}}\right|^{2}$ is distributed among the damped fermions states which build up a broad incoherent background.

For temperatures approaching $T_{\mathrm{c}}$, all bosons start to occupy only the lowest lying states such that $\tilde{E}_{\mathbf{q}} \sim 0$ which correspond $|\mathbf{q}| \leqslant q_{\text {crit }}$. Occupancy of the low momentum boson states is much larger than the Fermi function $f\left(\tilde{\varepsilon}_{\mathbf{q}-\mathbf{k}}\right)$ and the spectral function effectively becomes

$$
A^{\mathrm{F}}(\mathbf{k}, \omega) \simeq\left|\tilde{P}_{\mathbf{k}}\right|^{2} \delta\left(\omega-\tilde{\varepsilon}_{\mathbf{k}}\right)+\frac{\left|\tilde{r}_{\mathbf{k}, \mathbf{0}}\right|^{2}}{N} \sum_{|\mathbf{q}| \leqslant q_{\text {crit }}} b\left(\tilde{E}_{\mathbf{q}}\right) \delta\left(\omega+\tilde{\varepsilon}_{\mathbf{q}-\mathbf{k}}\right)
$$




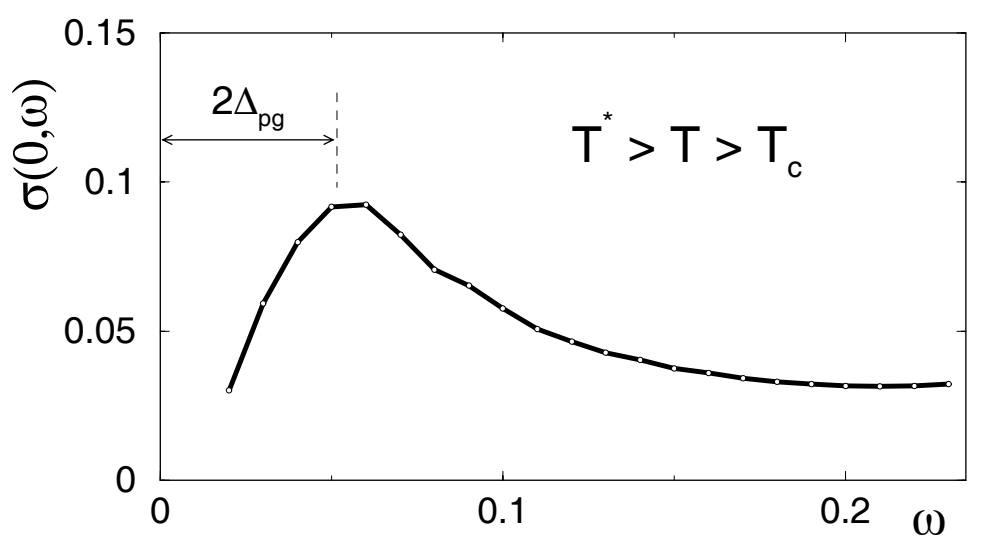

Figure 9. The optical conductivity $\sigma(\mathbf{q}, \omega)$ obtained in the London limit $\mathbf{q} \rightarrow \mathbf{0}$ for the pseudogap phase $T^{*}>T>T_{\mathrm{c}}$ using the boson fermion model. Energies $\omega, \Delta_{p g}$ are expressed in units of $W$ and the optical conductivity in units of $e^{2} / h$, where $h$ is the Planck constant.

where the second part gives rise to the appearance of the Lorentzian peak centered around $\omega=-\tilde{\varepsilon}_{-\mathbf{k}}$. This is a shadow branch of the Bogoliubov spectrum shown in figure 8. It is broadened because the fermion Cooper pairs can be present above $T_{\mathrm{c}}$ only as the damped quasiparticles [6]. Charge transport of the system will be affected by the excitations induced by the electromagnetic field between these two Bogoliubov branches which are split around the Fermi energy by the pseudogap $2 \Delta_{p g}[50]$.

The boson spectral function is expressed by the following general structure [6]

$$
\begin{aligned}
A^{\mathrm{B}}(\mathbf{q}, \omega)= & \left|\tilde{A}_{\mathbf{q}}\right|^{2} \delta\left(\omega-\tilde{E}_{\mathbf{q}}\right) \\
& +\frac{1}{N} \sum_{\mathbf{k}}\left[1-f\left(\tilde{\varepsilon}_{\mathbf{k}}\right)-f\left(\tilde{\varepsilon}_{\mathbf{q}-\mathbf{k}}\right)\right]\left|\tilde{B}_{\mathbf{q}, \mathbf{k}}\right|^{2} \delta\left(\omega-\tilde{\varepsilon}_{\mathbf{k}}-\tilde{\varepsilon}_{\mathbf{q}-\mathbf{k}}\right),
\end{aligned}
$$

where the first term represents the long-lived bosons with the renormalized energy $\tilde{E}_{\mathbf{q}}$ and the second contribution describes the incoherent background which is spread over a wide range of energies. Upon approaching $T_{\mathrm{c}}$ the incoherent background is partly expelled from the low energies and, therefore, the long-lived bosons increase their life-time. This feature is going to affect the charge transport.

The charge dynamics in the pseudogap region is thus determined by the following processes:

- the single particle fermion excitations between the lower and upper Bogoliubovlike branches which are separated near $\mathbf{k}_{\mathrm{F}}$ by $2 \Delta_{p g}(T)$ (one should keep in mind that the shadow branch fades away for temperatures exceeding $T^{*}$ when it merges the broad incoherent background),

- the single particle boson excitations which due to a gradual increase of the quasiparticle life-time (this issue is at some length discussed in [6]) contribute a non-diffusive type of transport. 
In figure 9 we present the optical conductivity calculated for the London limit $\mathbf{q} \rightarrow \mathbf{0}$ using the boson fermion model in the pseudogap regime $T^{*}>T>T_{\mathrm{c}}$. We notice a sizable reduction of the dynamic conductivity at frequencies below $2 \Delta_{p g}$. For comparison, let us remark that in the isotropic superconducting phase the absorption of external field quanta occurs above the energy threshold $\omega \geqslant 2 \Delta_{\text {sc }}$. For the pseudogap phase we obtain that the infrared absorption is not completely forbidden. However, it is strongly suppressed. This effect signifies the action of the pair fluctuations on the optical conductivity. Such a behavior has been indeed observed experimentally in the underdoped optimally doped cuprate superconductors from the measurements of the $c$-axis [52] and $a b$-plane [53] optical conductivity.

\section{Summary and conclusions}

We have studied the strongly interacting systems focusing on the calculation of the spectral and transport properties, such as: $d c$ conductivity of the strongly correlated alloys, the non-equilibrium conductance $G(V)$ via quantum dots coupled to the superconducting leads with various symmetries of the order parameter, and the optical conductivity $\sigma(\omega)$ for the high temperature superconductors in the pseudogap phase.

Our main findings are:

(i) the interplay of disorder and correlations manifests itself inter alia as a nonmonotonic dependence of $d c$ conductivity with respect to the Hubbard interaction $U$.

(ii) in a regime of the strong on-dot correlations, the tunnelling is dominated by the single particle current since the Andreev reflection processes are strongly suppressed. The calculated differential conductance and density of states exhibit the rich structure, which depends on a symmetry of the order parameter of superconductor.

(iii) the pseudogap remarkably effects the $a c$ charge transport of the system. At frequencies smaller than $2 \Delta_{p g}$ there occurs a partial suppression of the optical conductivity similar to that observed in a superconducting state. This is in agreement with the measurements for the underdoped and optimally doped high temperature superconductors above $T_{\mathrm{c}}$.

\section{Acknowledgements}

We would like to dedicate this work to the memory of Prof. Z.O. Gurskii. This study is partially supported by the KBN grant No. 2P03B 06225. 


\section{References}

1. Belitz D., Kirkpatrick T.R. // Rev. Mod. Phys., 1994, No. 66, p. 261.

2. Ng T.K., Lee P.A. // Phys. Rev., 1989, No. 61, 1768;

Glazman L.I., Raikh M.E. // Pis'ma Zh. Eksp. Teor. Fiz., 1988, No. 48, p. 378 [Engl. transl. JETP Lett., 1988, No. 47, p. 452];

Herschfield S., Davies J.H., Wilkins J.W. // Phys. Rev. Lett., 1991, No. 67, p. 3720.

3. Meir Y., Wingreen N.S., Lee P.A. // Phys. Rev. Lett., 1993, No. 70, p. 2601;

Wingreen N.S., Meir Y. // Phys. Rev. B, 1994, No. 49, p. 11040;

Meir Y., Wingreen N.S., Lee P.A. // Phys. Rev. Lett., 1991, No. 66, p. 3048.

4. Loss D., Di Vincezo D.P. // Phys. Rev. A, 1998, No. 57, p. 120.

5. Hartmann U., Wilhelm F.K. Decoherence of charge states in double quantum dots due to cotunneling. Preprint cond-mat/0208220.

6. Domański T., Ranninger J. // Phys. Rev. Lett., 2003, No. 91, p. 255301; // Phys. Rev. B, 2004, (submitted).

7. J. Ranninger, S. Robaszkiewicz // Physica B, 1985, No. 135, p. 468; S. Robaszkiewicz, R. Micnas, J. Ranninger // Phys. Rev. B, 1987, No. 36, p. 180; R. Friedberg, T.D. Lee // Phys. Rev. B, 1989, No. 40, p. 423; Ranninger J., Robin J.M. // Physica C, 1995, No. 253, p. 279; Ch.P. Enz // Phys. Rev. B, 1996, No. 54, p. 3589; V.B. Geshkenbein, L.B. Ioffe, Larkin A.I. // Phys. Rev. B, 1997, No. 55, p. 3173; E. Altman, A. Auerbach // Phys. Rev. B, 2003, No. 65, p. 104508; R. Micnas, S. Robaszkiewicz, BussmannHolder A. // Phys. Rev. B, 2002, No. 66, p. 104516; Ranninger J., Tripodi L. // Phys. Rev. B, 2003, No. 67, p. 174521, (and the references cited therein).

8. Kotliar G., Rückenstein A.E. // Phys. Rev. Lett., 1983, No. 57, p. 1362.

9. Lavagna M. // Phys. Rev. B, 1990, No. 41, p. 142.

10. Blackman J.A., Esterling D.M., Berk N.F. // Phys. Rev. B, 1971, No. 4, p. 2412.

11. Wysokiński K.I. // Phys. Rev. B, 1999, No. 60, p. 16376.

12. Wysokiński K.I. // J. Low Temp. Phys., 1999, No. 117, p. 235.

13. Michalik M., Wysokiński K.I., (in preparation).

14. Ferry D.K., Goodnick S.M. Transport in Nanostructures. Cambridge, Cambridge University Press, 1997.

15. Special issue on Single Charge Tunneling. // Z. Phys. B, 1991, vol. 85, No. 3.

16. Glazman L.I., Raikh M.E. // Pisma Zh. Eksp. Teor. Fiz., 1988, No. 47, p. 378.

17. Ng T.K., Lee P.A. // Phys. Rev. Lett., 1988, No. 61, p. 1768.

18. Kawabata A. // J. Phys. Soc. Jpn., 1991, No. 60, p. 3222.

19. Goldhaber-Gordon D., Shtrikman H., Mahalu D., Abush-Maggder D., Meirav U., Kastner M.A. // Nature, 1998, No. 391, p. 156.

20. Cronenwett S.M., Oosterkamp T.H., Kouwenhoven L.P. // Science, 1998, No. 281, p. 540 .

21. Schmid J., Weis J., Eberl K., von Klitzing K. // Physica B, 1998, No. 256-258, p. 182; Schmid J., Weis J., Eberl K., von Klitzing K. // Phys. Rev. Lett., 2000, No. 84, p. 5824.

22. Simmel F., Blick R.H., Kotthaus J.P., Wegsheider W., Bichler M. // Phys. Rev. Lett., 1999, No. 83, p. 804.

23. Sasaki S., De Franceschi S., Elzerman J.M., van der Wiel W.G., Eto M., Tarucha S., Kouwenhoven L.P. // Nature, 2000, No. 405, p. 764.

24. Andreev A.F. // Zh. Eksp. Teor. Fiz., 1964, No. 46, p. 1823; // Sov. Phys. JETP, 1964, No. 19, p. 1228. 
25. Lambert C.J., Raimondi R. // J. Phys. Condens. Matter, 1998, No. 10, p. 901.

26. Whan C.B., Orlando T.P. // Phys. Rev. B, 1996, No. 54, p. R5255.

27. Levy Yeyati A., Cuevas J.C., López-Dávalos A., Martín-Rodero A. // Phys. Rev. B, 1997, No. 55, p. R6137.

28. Kang K. // Phys. Rev. B, 1998, No. 57, p. 11891.

29. Ivanov T.I. // Phys. Rev. B, 1999, No. 59, p. 169.

30. Krawiec M., Wysokiński K.I. // Acta. Phys. Polon. A, 2000, No. 97, p. 197.

31. Avishai Y., Golub A., Zaikin A.D. // Phys. Rev. B, 2003, No. 67, p. R041301.

32. Ishizaka S., Sone J., Ando T. // Phys. Rev. B, 1995, No. 52, p. 8358.

33. Liu S.Y., Lei X.L. Preprint cond-mat/0312148.

34. Choi M.-S., Lee M., Kang K., Belzig W. Preprint cond-mat/0312271.

35. Ralph D.C., Black C.T., Tinkham M. // Phys. Rev. Lett., 1995, No. 74, p. 3241; Black C.T., Ralph D.C., Tinkham M. // ibid., 1996, No. 76, p. 688.

36. Buitelaar M.R., Nussbaumer T., Shönenberger C. // Phys. Rev. Lett., 2002, No. 89, p. 256801.

37. Anderson P.W. // Phys. Rev., 1961, No. 124, p. 41.

38. Barnes S.E. // J. Phys., 1976, No. F6, p. 1375; // J. Phys., 1977, No. F7, p. 2631.

39. Coleman P. // Phys. Rev. B, 1984, No. 29, p. 3035.

40. Keldysh L.V. // Zh. Eksp. Teor. Fiz., 1965, No. 47, p. 1515.

41. Niu C., Lin D.L., T.-H. Lin // J. Phys. Condens. Matt., 1999, No. 11, p. 1511.

42. Le Guillou J.C., Ragoucy E. // Phys. Rev. B, 1995, No. 52, p. 2403.

43. Krawiec M., Wysokiński K.I. // Phys. Rev. B, 1999, No. 60, p. 9500.

44. Krawiec M., Wysokiński K.I. // Solid State Commun., 2000, No. 115, p. 141.

45. Borkowski L., Hirschfeld P.J. // J. Low Temp. Phys., 1994, No. 96, p. 185.

46. Withoff D., Fradkin E. // Phys. Rev. Lett., 1990, No. 64, p. 1835.

47. Corson J., Mallozzi R., Orenstein J., Eckstein J.N., Bozovic I. // Nature, 1999, No. 398, p. 221; Xu Z.A., Ong N.P., Wang Y., Takeshita T., Uchida S. // Nature, 2000, No. 406, p. 486.

48. Mahan G.D. Many Particle Physics. New York, Plenum Press, 1981.

49. Kostyrko T., Ranninger J. // Phys. Rev. B, 1996, No. 54, p. 13105.

50. Domański T., Ranninger J. // Phys. Rev. B, 2001, No. 63, p. 134505; // Physica C, 2003, No. 387, p. 77.

51. Wegner F. // Ann. Physik (Leipzig), 1994, No. 3, p. 77; // Adv. Solid State Phys., 2000, No. 40, p. 113; // Phys. Rep., 2001, No. 348, p. 77.

52. Hwang J., Timusk T., Gu G.D. // Nature, 2004, No. 427, p. 714; Homes C.C., Timusk T., Liang R., Bonn D.A., Hardy W.N. // Phys. Rev. Lett., 1993, No. 71, p. 1645.

53. Homes C.C., Dordevic S.V., Bonn D.A., Liang R., Hardy W.N. // Phys. Rev. B, 2004, No. 69, p. 024514; Molegraaf H.J.A., Presura C., van der Marel D., Kes P.H., Li M. // Science, 2002, No. 295, p. 2239; Santander-Syro A.F., Lobo R.P.S.M., Bontemps N., Konstaninovic Z., Li Z.Z., Raffy H. // Phys. Rev. Lett., 2002, No. 88, p. 097005. 


\title{
Властивості переносу в сильно корельованих системах
}

\author{
Т.Доманьскі, М.Кравєц, М.Міхалік, К.І.Високіньскі \\ Інститут фізики, Університет М.Кюрі-Склодовської, \\ 20-031 Люблін, Польща
}

Отримано 12 березня 2004 р., в остаточному вигляді 6 травня 2004 р.

Властивості переносу для різних систем вивчаються тут у контексті трьох різних моделей: 1) невпорядкована модель Габарда, що застосовується до корельованих бінарних сплавів із загальним безладом, 2) модель Андерсона для опису фізики Кондо у квантовій точці, під'єднаній до зовнішніх надпровідників, та 3) модель РанінгераРобашкєвіча, що застосовується до вивчення оптичних властивостей системи з преутвореними електронними парами вище температури переходу в надпровідний стан. Ми розраховуємо густину станів, теплоємність, співвідношення Вільсона та провідність для корельованого бінарного сплаву з недіагональним безладом. Ми досліджуємо умови, при яких пік Кондо появляється в густині станів та в провідності квантової точки, приєднаної до зовнішніх надпровідників. Ми аналізуємо вплив псевдощілини на оптичні спектри у високотемпературних надпровідниках, що описуються бозон-ферміонною моделлю.

Ключові слова: наближення когерентного потенціалу, допоміжні бозони, нерівноважний перенос, квантова точка, неперервне перетворення, псевдощілина

PACS: 71.10.-w, 72.10.-d, 73.23.-b, 74.20.-z 